\title{
Efficacy of intradiscal hepatocyte growth factor injection for the treatment of intervertebral disc degeneration
}

\author{
FEI ZOU, JIANYUAN JIANG, FEIZHOU LU, XIAOSHENG MA, \\ XINLEI XIA, LIXUN WANG and HONGLI WANG \\ Department of Orthopaedics, Huashan Hospital, Fudan University, Shanghai 200040, P.R. China
}

Received January 18, 2013; Accepted April 22, 2013

DOI: $10.3892 / \mathrm{mmr} .2013 .1450$

\begin{abstract}
A poor nutritional supply to the cells of the avascular intervertebral discs, caused by dehydration of the extracellular matrix, is a major cause of intervertebral disc degeneration (IDD). Since hepatocyte growth factor (HGF) has been shown to exert antifibrotic effects, we hypothesized that HGF treatment may be capable of retarding IDD. The present study aimed to evaluate the efficacy of HGF treatment in retarding IDD in a rat tail model of disc degeneration. The disc degeneration models were induced by needle puncture of the rat tail discs. Four weeks following needle puncture, a triblock poly(lactide-co-glycolide)-poly(ethyleneglycol)poly(lactide-co-glycolide; PLGA-PEG-PLGA) polymer gel loaded with HGF or the gel alone was injected into rat tail discs. The efficacy of HGF in retarding IDD was assessed by magnetic resonance imaging (MRI), histological and immunohistochemical evaluation of the type I collagen, type II collagen and bone morphogenetic protein-2 (BMP-2) expression levels. Following injection of the HGF-loaded gel into the nucleus pulposus (NP), a significant trend towards an increase in $\mathrm{T} 2$ signal intensity $(\mathrm{P}=0.028)$, type II collagen staining in the NP and the number of BMP-2-positive cells in the annulus fibrosus was observed. In addition, the results demonstrated a significant trend towards a decrease in the histological score $(\mathrm{P}=0.025)$ and type I collagen staining in the NP compared with segments treated with the gel alone, following the induction of disc degeneration by stab injury. Following treatment with HGF, a tendency for the level of disc height to be maintained was also observed (no statistical significance). By MRI, histological and immunohistochemical evaluation, the present study demonstrated that HGF-loaded PLGA-PEG-PLGA gel was able to retard disc degeneration when injected into the degenerative discs of rat tail models.
\end{abstract}

Correspondence to: Professor Jianyuan Jiang, Department of Orthopaedics, Huashan Hospital, Fudan University, No. 12 Wulumuqizhong Road, Shanghai 200040, P.R. China

E-mail: jjy@fudanspine.com

Key words: hepatocyte growth factor, intervertebral disc, degeneration, rat model

\section{Introduction}

Intervertebral disc degeneration (IDD) is one of the most common clinical entities and the leading etiological contributor to low-back pain and other disc disorders (1). The progression of disc degeneration is associated with a significant decrease in the levels of proteoglycans (PGs) and water, as well as an increase in the levels of type I collagen in the nucleus pulposus (NP) with the simultaneous decrease in type II collagen levels (2-4). Thus, a poor nutritional environment as a result of dehydration in the discs is a major cause of degeneration of the intervertebral discs. As a result of their ease of use, minimally invasive properties and efficient correction of metabolic derangement, protein growth factor injections administered into degenerative discs have become an alternative treatment strategy to conservative therapy and reconstructive surgery for the treatment of symptomatic IDD (5).

Hepatocyte growth factor (HGF) was initially identified as a mitogen of hepatocytes and promotes the regeneration and repair of the kidney, lung and several other tissues (6-8). In terms of its clinical application, human HGF plasmid DNA has been used in the treatment of critical limb ischemia $(9,10)$. Given its therapeutic effects and good safety profile observed in animal experiments and clinical trials, we hypothesized that HGF therapy is capable of retarding IDD. To the best of our knowledge, no studies examining the therapeutic effects of HGF in degenerative discs have been previously conducted. In the present study, a rat tail IDD model induced by needle puncture was used due to its convenience, minimally invasive properties and cost-effectiveness (11). The triblock poly(lactide-co-glycolide)-poly(ethyleneglycol)poly(lactide-co-glycolide; PLGA-PEG-PLGA) polymer was used as the drug vehicle due to its optimal properties, including biodegradability, thermosensitivity and allowing a prolonged duration of drug exposure. We performed magnetic resonance imaging (MRI), histological and immunohistochemical assessments in a rat tail IDD model, which demonstrated that HGF retards the process of IDD by stimulating antifibrosis in degenerative discs.

\section{Materials and methods}

Reagents and antibodies. Recombinant human HGF was purchased from HumanZyme Inc. (Chicago, IL, USA). Rabbit 
polyclonal type I and type II collagen, bone morphogenetic protein-2 (BMP-2) and secondary goat polyclonal fluorescein isothiocyanate (FITC)-conjugated antibodies were purchased from Abcam (Cambridge, MA, USA). PLGA-PEG-PLGA was synthesized at our laboratory (Fudan University, Shanghai, China).

Animals. Thirty male Sprague-Dawley rats (each weighing 200-250 g) were used in this study. The animals were housed with free access to commercial rodent chow and water. The temperature was maintained at $24^{\circ} \mathrm{C}$ and the light schedule consisted of $12 \mathrm{~h}$ of daylight starting at 8:00 a.m. and $12 \mathrm{~h}$ of darkness starting at 8:00 p.m. The humidity was maintained at $50 \%$. All of the experiments were performed according to the guidelines for the ethical treatment of animals approved by the Laboratory Animal Ethics Committee of Fudan University (Shanghai, China).

Surgical procedure. The rat tail segments were divided into 4 groups: the sham-operated control segments, stab injuryonly segments, gel alone-treated segments and HGF-treated segments. The animals were anesthetized using a combination of $10 \mathrm{mg} / \mathrm{kg}$ xylazine and $60 \mathrm{mg} / \mathrm{kg}$ ketamine hydrochloride administered intraperitoneally. A total of 10 rat tails were prepared for drug treatment. For the MRI assessment, the caudal segments 5/6 and 7/8 from the same rat tail were interchangeably used for the gel alone treatment and HGF treatment. After identifying the level of the caudal segments by fluoroscopy, a puncture was made parallel to the endplates through the annulus fibrosus (AF) into the NP by a syringe needle (21-gauge) (12). The needle was then rotated by $180^{\circ}$ and held for $5 \mathrm{sec}$. During the puncture, fluoroscopy was used to ensure that the needle reached the center of the NP (11). The caudal segments $6 / 7$ were left undisturbed as contrast segments for MRI. For the stab injury-only segments, an additional 10 rat tails underwent the same surgical procedure randomly at the level of caudal segments $5 / 6$ or $7 / 8$ in order to correspond to the drug-treated segments described above. The remaining 10 rat tails were used to prepare the sham-operated control segments, for which only a small incision was made in the tail skin.

Four weeks following needle puncture, the PLGA-PEGPLGA gel loaded with HGF or the gel alone was slowly injected into the NP of the respective caudal segments of the rat tail. Recombinant human HGF (HumanZyme Inc.) was added to a $30 \%$ (w/v) aqueous solution of PLGA-PEG-PLGA and homogenized at 9,600 x g for $30 \mathrm{sec}$ at room temperature to form a homogeneous clear solution (HGF, $5 \mu \mathrm{g} / \mathrm{ml}$ ) (13). To avoid injury leading to disc degeneration, a micro-syringe attached to a 31-gauge needle (Hamilton Bonaduz AG, Bonaduz, Switzerland) was used and the injection volume was $2 \mu \mathrm{l}(14,15)$.

MRI evaluation. The effect of stab injury and HGF treatment on disc degeneration was evaluated using a Siemens Trio Tim 3.0T MR scanner (Siemens Medical Solutions, Erlangen, Germany) at 2 and 4 weeks following stab injury, and at 2 and 4 weeks following drug injection. The animals were laid prone and the tails were straightened in the MR scanner. Serial T2-weighted sagittal and transverse images covering the entire experimental disc area were obtained using the following parameter settings: repetition time, $6150 \mathrm{msec}$; echo time,
$80 \mathrm{msec}$; field of view, $60 \times 35 \mathrm{~mm}$ and slice thickness, $0.8 \mathrm{~mm}$. T2-weighted signal intensity and disc height were measured using the Image J software (The National Institutes of Health, Bethesda, MD, USA). Image assessments were conducted by two independent, blinded and experienced observers. The data were presented as the mean of the two evaluations.

Histological evaluation. At 4 weeks following drug injection, all of the rats were euthanized and the segments were harvested for histological examination. The discs were fixed and decalcified, and then processed for paraffin embedding and sectioning. Sagittal sections $(5 \mu \mathrm{m})$ were obtained and stained with hematoxylin and eosin (H\&E). Histological images were analyzed using the Olympus BX51 microscope (Olympus, Center Valley, PA, USA). The extent of disc degeneration was graded using a semi-quantitative method as described previously by Masuda et al (16). The assessment was conducted by two independent, blinded and experienced observers. The data were presented as the mean of the two evaluations.

Immunohistochemical examination. For the immunohistochemical examination, the sections were stained with rabbit anti-type I and type II collagen, and anti-BMP-2 antibodies (Abcam). Paraffin-embedded sections were deparaffinized and rehydrated, immersed in a retrieval solution $(10 \mathrm{mmol} / \mathrm{l}$ citrate, $\mathrm{pH}$ 6.0) and then placed in a microwave for $10 \mathrm{~min}$. Endogenous peroxidase activity was blocked using $3 \%$ hydrogen peroxide for $10 \mathrm{~min}$. Non-specific binding sites were blocked using $1 \%$ bovine serum albumin for $30 \mathrm{~min}$ at room temperature. The sections were incubated with the primary antibody at $4^{\circ} \mathrm{C}$ overnight and then with the FITC-conjugated secondary antibody for $30 \mathrm{~min}$ at room temperature. The diaminobenzidine detection method was used to visualize the BMP-2 protein expression (11) and the sections were counterstained using hematoxylin.

Statistical analysis. The SPSS version 17.0 software (SPSS, Inc., Chicago, IL, USA) was used for all statistical analyses. Data were expressed as the mean \pm standard error. The Kruskal-Wallis test was used to analyze the differences in the T2 signal intensities and in the histological scores of the discs. One-way ANOVA was used to analyze the differences in disc height. $\mathrm{P}<0.05$ was considered to indicate a statistically significant result.

\section{Results}

MRI assessment. The T2 signal intensity was decreased in the gel alone-treated and HGF-treated segments at 2 and 4 weeks following stab injury. However, the signal intensity in the HGF-treated segments was increased at 4 weeks following drug injection compared with the gel alone-treated segments, and the difference was statistically significant $(\mathrm{P}=0.028$; Fig. 1A and B). The degree of disc height reduction in the HGF-treated segments was smaller compared with the gel alone-treated segments at 2 and 4 weeks following drug injection; however, the difference was not statistically significant (P>0.05; Fig. 1C).

Histological changes and scores. H\&E staining demonstrated an intact circumferential AF in the sham-operated 
A

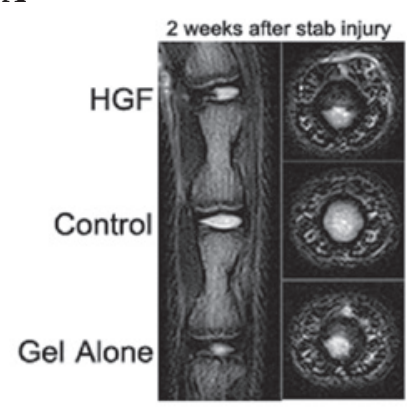

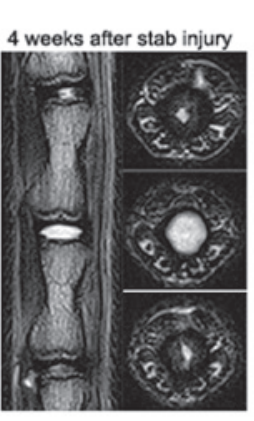

B

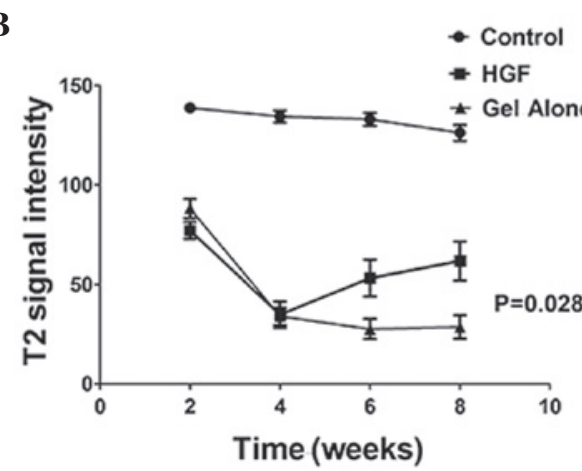

6 weeks after stab injury $\quad 8$ weeks after stab injury

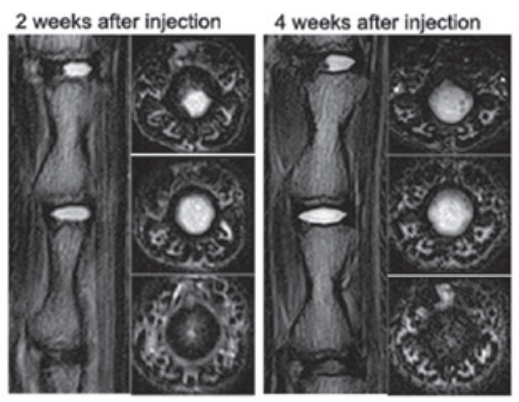

C

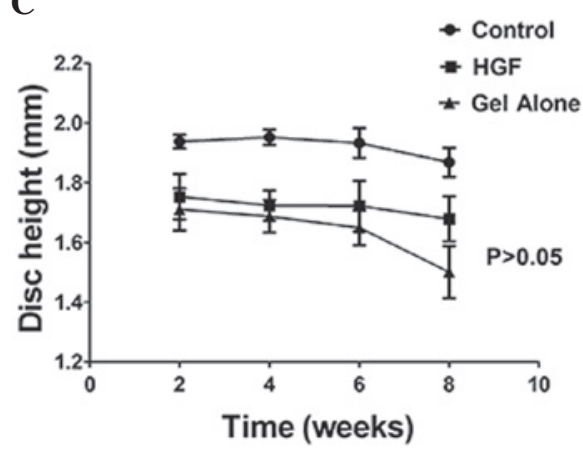

Figure 1. (A) T2-weighted magnetic resonance images. (B) T2-weighted signal intensities ( $\mathrm{P}=0.028$, HGF vs. gel alone; mean $\pm \mathrm{SEM}$; $\mathrm{n}=5$ ). (C) Disc height ( $\mathrm{P}>0.05$, HGF vs. gel alone; mean $\pm \mathrm{SEM} ; \mathrm{n}=5$ ) of the drug-treated rat tail segments. HGF, hepatocyte growth factor.

control segments and the border between the AF and the NP was clear. The NP occupied the majority of the disc area. In the stab injury-only and gel alone-treated segments, ruptured and serpentine-patterned fibers were observed in the AF. Furthermore, the border between the AF and NP was interrupted and unclear, and exhibited features of severe degeneration. However, the AF and the border between the AF and the NP in the HGF-treated segments appeared more regular compared with those observed in the stab-injury only and gel alone-treated segments (Fig. 2A). Statistical analysis demonstrated that the histological score of the HGF-treated segments was significantly lower compared with the gel alonetreated segments $(\mathrm{P}=0.025$; Fig. 2B).

Immunohistochemical evaluation. Immunofluorescence studies revealed that the staining for type II collagen was stronger in the HGF-treated segments compared with the other experimental segments. By contrast, the staining was weaker in the stab-injury only and gel alone-treated segments when compared with the sham-operated control segments. By contrast, type I collagen staining appeared strongly positive in the stab-injury only and gel alone-treated segments when compared with the sham-operated control segments. However, in the HGF-treated segments, type I collagen was almost exclusively observed in the outer layer of the AF (Fig. 3).

The expression levels of BMP-2 were detected by immunohistochemical staining (Fig. 4). The BMP-2 expression levels were negligible in the sham-operated control segments. Conversely, the BMP-2 expression levels were notably increased in the AF cells of the HGF-treated segments. By contrast, only the disrupted border in the AF demonstrated a strong positive staining for BMP-2 expression in the stab injury-only and gel alone-treated segments (Fig. 4).

\section{Discussion}

When evaluating the degree of disc degeneration, the two most important clinical parameters to consider are the values of T2-weighted signal intensity and disc height, which are measured by MRI. Therefore, we evaluated MR scans in order to assess disc degeneration intravitally and continuously in vivo. The signal intensity of the $\mathrm{T} 2$-weighted images and disc height on the MR scans represented the water content and the NP volume in the intervertebral discs, and the loss of water content and NP volume were associated with a reduction in the levels of $\mathrm{PGs}$ in the NP, contributing to the decrease in the T2-weighted signal intensity and disc height. Thus, disc regeneration leads to an increased signal intensity of the T2-weighted image and disc height $(17,18)$. Our results demonstrated that degenerative changes in discs induced by stab injury were recovered following HGF injection. The T2-weighted signal intensity of the HGF-treated segments was significantly stronger than that of the gel alone-treated segments at 4 weeks following drug injection. Although the differences in disc height between the HGF-treated and gel alone-treated segments was not statistically significant following drug injection, our results demonstrated a trend towards HGF treatment retarding the reduction of disc height.

During degeneration, discs become less elastic, consequently inhibiting their ability to absorb and dissipate spinal forces. The increase in type I collagen levels along with the corresponding decrease in type II collagen and PG levels in the disc matrix has been identified as a factor that causes the poor nutritional environment and inferior mechanical properties of degenerated discs (19). In samples of human lumbar intervertebral discs, type II collagen levels were demonstrated to be present at normal levels in the NP and in the inner layer of the AF; however, the 
A

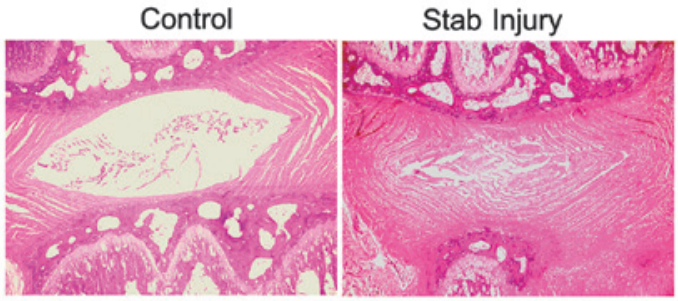

Gel alone

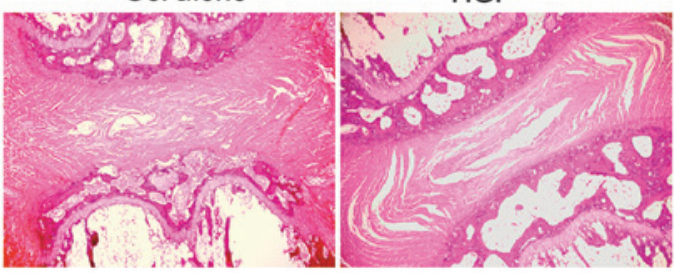

B

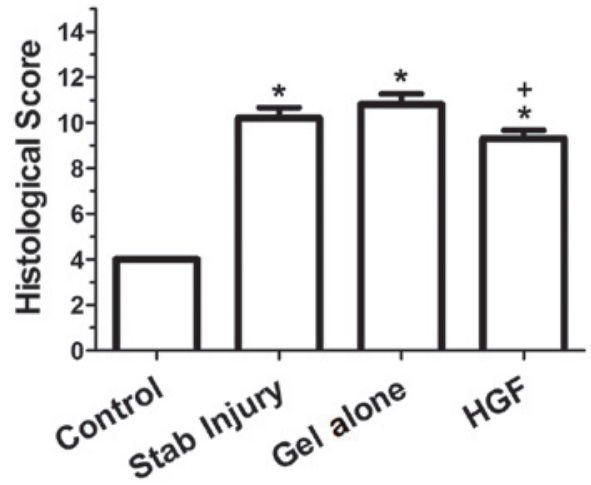

Figure 2. (A) H\&E staining (magnification, $\mathrm{x} 40$ ). (B) Histological scores $\left({ }^{*} \mathrm{P}<0.01\right.$ vs. control, ${ }^{+} \mathrm{P}<0.05$ vs. gel alone; mean \pm SEM; $\left.\mathrm{n}=10\right)$ of disc samples from different experimental segments at 4 weeks following drug injection. H\&E, hematoxylin and eosin; HGF, hepatocyte growth factor.

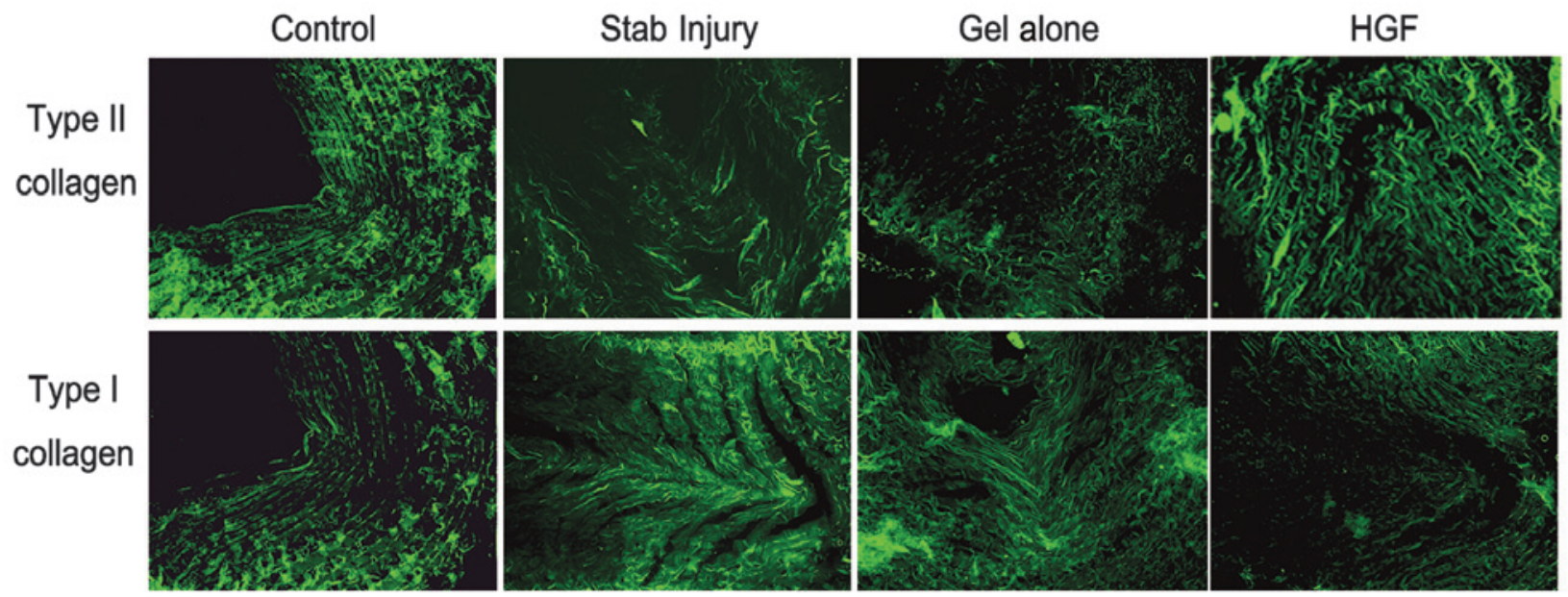

Figure 3. Type II and I collagen immunofluorescence of disc samples from different experimental segments at 4 weeks following drug injection (magnification, x200). HGF, hepatocyte growth factor.
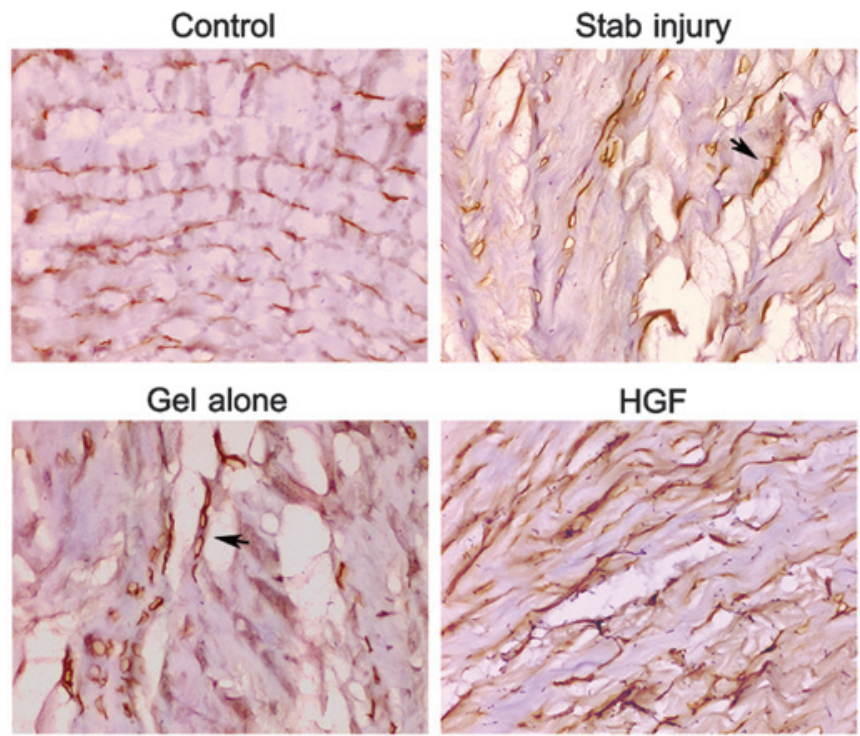

Figure 4. BMP-2 immunohistochemical staining in the annulus fibrosus of disc samples from different experimental segments at 4 weeks following drug injection (magnification, x400). The arrows indicate the BMP-2-positive cells near the disrupted border. HGF, hepatocyte growth factor; BMP-2, bone morphogenetic protein-2. 
levels were reduced in advanced degenerative discs. In addition, type I collagen levels were demonstrated to be present at normal levels in the AF and degenerative NP (2). In a rabbit annular stab-injury model, aggrecan and type IIa collagen mRNA levels were decreased and were unable to be restored to normal levels, whereas type Ia collagen mRNA levels gradually increased throughout the course of degeneration (20). Our results demonstrated that in degenerative discs treated with HGF, the levels of type I collagen synthesis in the NP were suppressed; however, changes in the levels of synthesis of intrinsic type I collagen in the outer layer of the AF were not observed, and type II collagen levels in the NP were restored. Our results indicate that HGF treatment provides an optimum method to maintain the normal ECM components and mechanical properties of discs.

The expression of endogenous growth factors in cells is required for degenerative disc repair. In degenerative disc models, the decreased expression of the BMP-2 gene may represent a failed reparative response to injury $(20,21)$. In an external-compression degenerative disc model, immunohistochemical evaluation of the compressed discs demonstrated a loss of the annular architecture and a significant reduction in the number of BMP-2-positive cells, whereas gene expression analysis showed a significant upregulation of BMP-2 (22). In a rabbit annular stab-injury model, there was a relative decrease in BMP-2 mRNA levels, followed by a consequent increase close to the preoperative levels (20). In a prospective, randomized controlled animal study, injection of the BMP-2 gene delayed degenerative changes in a rabbit disc puncture model (23). Our results demonstrated that the number of BMP-2-positive cells in the AF increased following treatment with HGF, whereas a strong positive staining of BMP-2 was only observed in cells at the disrupted border in the AF of stab-injury only and gel alone-treated segments, suggesting that HGF promotes BMP-2-induced repair in degenerative discs. In addition, an in vitro study using rat disc cells demonstrated the effect of BMP-2 on type II collagen and aggrecan mRNA expression levels (24). These findings suggest that the upregulation of BMP-2 in HGF-treated segments may have stimulated type II collagen and aggrecan synthesis observed in our study. However, the correlation between HGF and BMPs, and the precise role of each protein in degenerative disc repair requires further elucidation.

In conclusion, the present study has demonstrated that HGF-loaded PLGA-PEG-PLGA gel injected into the degenerative discs of rat tail models retards disc degeneration, as revealed by MRI, histological and immunohistochemical evaluation. The study provides an alternative biological method to achieve antifibrosis in the NP to induce disc repair. Considering the differences between the rat tail spine and the human lumbar spine, additional studies using a different animal model that closely resembles the characteristics of the human lumbar spine are required in order to evaluate the biological effects of HGF in degenerative discs.

\section{References}

1. Andersson GB: Epidemiological features of chronic low-back pain. Lancet 354: 581-585, 1999.

2. Nerlich AG, Boos N, Wiest I and Aebi M: Immunolocalization of major interstitial collagen types in human lumbar intervertebral discs of various ages. Virchows Arch 432: 67-76, 1998.
3. Zhao CQ, Wang LM, Jiang LS and Dai LY: The cell biology of intervertebral disc aging and degeneration. Ageing Res Rev 6: 247-261, 2007.

4. Bernick S, Walker JM and Paule WJ: Age changes to the anulus fibrosus in human intervertebral discs. Spine (Phila Pa 1976) 16: 520-524, 1991.

5. Masuda K, Oegema TR Jr and An HS: Growth factors and treatment of intervertebral disc degeneration. Spine (Phila Pa 1976) 29: 2757-2769, 2004

6. Matsumoto K and Nakamura T: Hepatocyte growth factor: molecular structure, roles in liver regeneration, and other biological functions. Crit Rev Oncog 3: 27-54, 1992.

7. Mizuno S and Nakamura T: Hepatocyte growth factor: a regenerative drug for acute hepatitis and liver cirrhosis. Regen Med 2: 161-170, 2007.

8. Mizuno S, Matsumoto K and Nakamura T: HGF as a renotrophic and anti-fibrotic regulator in chronic renal disease. Front Biosci 13: 7072-7086, 2008.

9. Shigematsu H, Yasuda K, Iwai T, et al: Randomized, doubleblind, placebo-controlled clinical trial of hepatocyte growth factor plasmid for critical limb ischemia. Gene Ther 17: 1152-1161, 2010.

10. Powell RJ, Simons M, Mendelsohn FO, et al: Results of a double-blind, placebo-controlled study to assess the safety of intramuscular injection of hepatocyte growth factor plasmid to improve limb perfusion in patients with critical limb ischemia. Circulation 118: 58-65, 2008.

11. Zhang H, La Marca F, Hollister SJ, Goldstein SA and Lin CY: Developing consistently reproducible intervertebral disc degeneration at rat caudal spine by using needle puncture. J Neurosurg Spine 10: 522-530, 2009.

12. Hsieh AH, Hwang D, Ryan DA, Freeman AK and Kim H: Degenerative anular changes induced by puncture are associated with insufficiency of disc biomechanical function. Spine (Phila Pa 1976) 34: 998-1005, 2009.

13. Chen S and Singh J: Controlled release of growth hormone from thermosensitive triblock copolymer systems: In vitro and in vivo evaluation. Int J Pharm 352: 58-65, 2008.

14. Jeong JH, Lee JH, Jin ES, Min JK, Jeon SR and Choi KH: Regeneration of intervertebral discs in a rat disc degeneration model by implanted adipose-tissue-derived stromal cells. Acta Neurochir (Wien) 152: 1771-1777, 2010.

15. Mao HJ, Chen QX, Han B, et al: The effect of injection volume on disc degeneration in a rat tail model. Spine (Phila Pa 1976) 36: E1062-E1069, 2011.

16. Masuda K, Aota Y, Muehleman C, et al: A novel rabbit model of mild, reproducible disc degeneration by an anulus needle puncture: correlation between the degree of disc injury and radiological and histological appearances of disc degeneration. Spine (Phila Pa 1976) 30: 5-14, 2005.

17. Roberts N, Gratin C and Whitehouse GH: MRI analysis of lumbar intervertebral disc height in young and older populations. J Magn Reson Imaging 7: 880-886, 1997.

18. Schiebler ML, Camerino VJ, Fallon MD, Zlatkin MB, Grenier N and Kressel HY: In vivo and ex vivo magnetic resonance imaging evaluation of early disc degeneration with histopathologic correlation. Spine (Phila Pa 1976) 16: 635-640, 1991.

19. Ruan DK, Xin H, Zhang C, et al: Experimental intervertebral disc regeneration with tissue-engineered composite in a canine model. Tissue Engineering Part A 16: 2381-2389, 2010.

20. Sobajima S, Shimer AL, Chadderdon RC, et al: Quantitative analysis of gene expression in a rabbit model of intervertebral disc degeneration by real-time polymerase chain reaction. Spine J 5: 14-23, 2005.

21. Sowa G, Vadalà G, Studer R, et al: Characterization of intervertebral disc aging: longitudinal analysis of a rabbit model by magnetic resonance imaging, histology, and gene expression. Spine (Phila Pa 1976) 33: 1821-1828, 2008.

22. Guehring T, Omlor GW, Lorenz H, et al: Stimulation of gene expression and loss of anular architecture caused by experimental disc degeneration - an in vivo animal study. Spine (Phila Pa 1976) 30: 2510-2515, 2005.

23. Leckie SK, Bechara BP, Hartman RA, et al: Injection of AAV2-BMP2 and AAV2-TIMP1 into the nucleus pulposus slows the course of intervertebral disc degeneration in an in vivo rabbit model. Spine J 12: 7-20, 2012.

24. Li J, Yoon ST and Hutton WC: Effect of bone morphogenetic protein-2 (BMP-2) on matrix production, other BMPs, and BMP receptors in rat intervertebral disc cells. J Spinal Disord Tech 17: $423-428,2004$ 